\title{
Giant leiomyoma of the gastroesophageal junction: technique and results of endoscopic full-thickness resection
}

This article was published in the following Dove Press journal:

Clinical and Experimental Gastroenterology

29 November 2011

Number of times this article has been viewed

\section{Davide Bona \\ Alberto Aiolfi \\ Stefano Siboni \\ Daniele Bernardi \\ Luigi Bonavina}

Department of Surgery, IRCCS

Policlinico San Donato, University of Milano School of Medicine,

Milano, Italy
Correspondence: Luigi Bonavina

Dipartimento Universitario di Chirurgia, IRCCS Policlinico San Donato, Piazza

E Malan 2, 20097 San Donato Milanese, Milano, Italy

$\mathrm{Tel}+390252774621$

Fax +390252774622

Email luigi.bonavina@unimi.it

\begin{abstract}
Four consecutive patients with a giant leiomyoma originating from the posterior aspect of the gastroesophageal junction were treated with full-thickness endoscopic retroflex dissection. A complete removal of the lesion was obtained in all cases. No complications were observed except for some degree of air filtration causing symptomatic pneumoperitoneum in one patient. Retroflex endoscopic full-thickness resection of giant leiomyoma at the gastroesophageal junction is feasible and safe.
\end{abstract}

Keywords: gastroesophageal junction, leiomyoma, endoscopic submucosal dissection, pneumoperitoneum

\section{Background}

Leiomyoma is the most common smooth muscle tumor of the stomach and represents $80 \%$ of all gastric stromal tumors. About one-third of these neoplasms are located at the gastroesophageal junction and are often asymptomatic or minimally symptomatic. ${ }^{1-3}$ Surgical removal can be required when the lesion is large or rapidly increasing in size. ${ }^{4}$ Resection of leiomyoma of the gastroesophageal junction has been traditionally performed via laparotomy. ${ }^{5}$ When the tumor originates from the anterior wall of the cardia and exhibits an endophytic growth pattern, a hybrid approach through transgastric laparoscopy combined with endoscopic assistance has been shown to be safe and feasible. ${ }^{6-8}$ It is particularly challenging to treat submucosal lesions located at the posterior aspect of the gastroesophageal junction with this combined approach because of a high risk of causing deformity, stricture, or leakage. Park et al ${ }^{9}$ first proposed a new endoscopic approach for enucleation of upper gastrointestinal submucosal tumors by using an insulated-tip electrosurgical knife. We present our experience with full-thickness endoscopic resection of giant leiomyomas originating from the posterior side of the gastroesophageal junction.

\section{Methods}

Between October 2008 and December 2010, four consecutive patients with leiomyoma of the gastroesophageal junction were treated endoscopically at our institution. The standard preoperative examinations included barium swallow study, upper gastrointestinal endoscopy, endoscopic ultrasonography, and thoracoabdominal computed tomography scan. No endoscopic biopsies were performed. One patient underwent esophageal manometry and 24-hour esophageal $\mathrm{pH}$ monitoring because of intermittent complaints of retrosternal heartburn and dysphagia. 
All patients were clearly informed about the planned procedure, including the possibility of switching to a laparoscopic/laparotomic approach, depending on the occurrence of intraoperative technical difficulties. Intravenous cefazoline $(2 \mathrm{~g})$ was given to all patients before the procedure.

The procedure was performed in the operating room, under general anesthesia, with orotracheal intubation and with the patient in the supine position. A standard $9 \mathrm{~mm}$ endoscope with a soft, transparent hood attached to its tip was advanced through an overtube into the stomach and then retroflexed (Figure 1). After submucosal injection of $10 \mathrm{~mL}$ of diluted epinephrine, an incision of the gastric mucosa overlying the mass was carried out with a retroflexed approach by using an insulated-tip diathermic electrosurgical knife (IT-Knife 2; Olympus, Tokyo, Japan) and a hook knife (Olympus Optical, Tokyo, Japan). In all cases the dissection started along the lower border of the lesion and then was extended circumferentially (Figure 2). Once the submucosal layer was reached, the tumor was gradually enucleated and then extracted from the mouth with an endocatch. Finally, the gastric mucosal edges were approximated under mild suction and closed with three to five metallic clips (Boston Scientific, Natick, MA) (Figures 3 and 4).

\section{Results}

There were three females and one male with a mean age of 44 years (range 36-58 years). None of them had previous gastric surgery or significant comorbidity. The mean duration of symptoms was 10 months (range 4-18 months). The predominant symptom was reflux-like

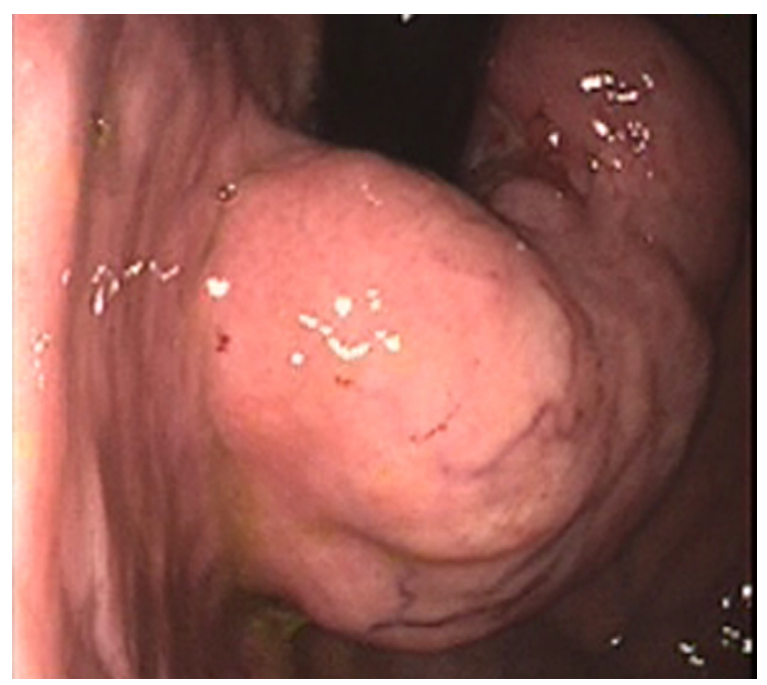

Figure I Retroflexed endoscopic view of a giant leiomyoma of the gastroesophageal junction.

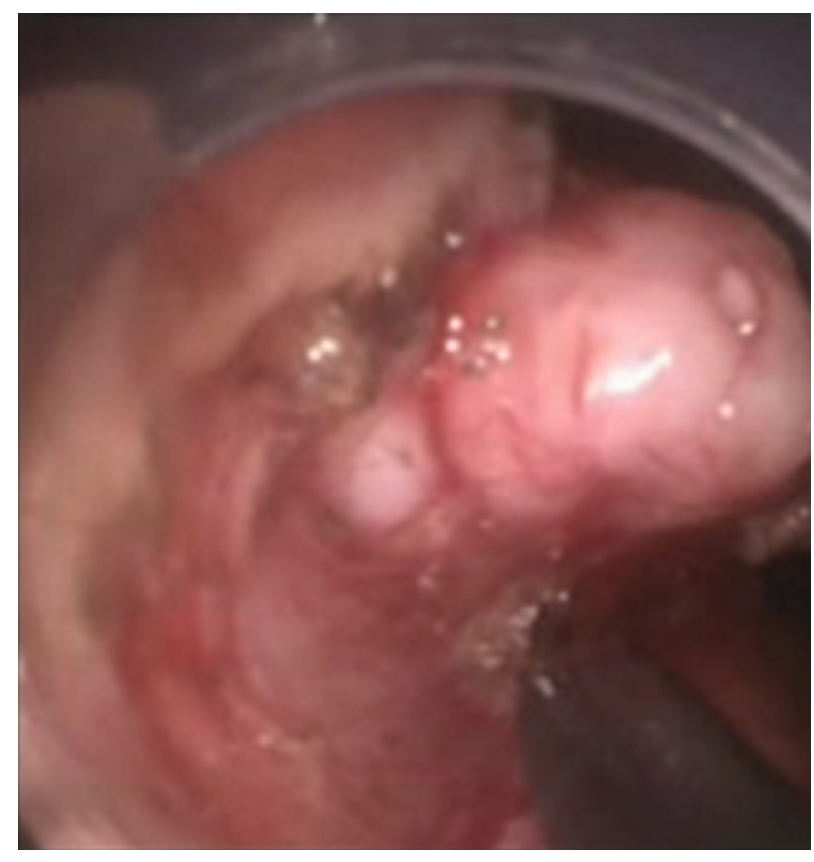

Figure 2 Submucosal dissection of the leiomyoma started along the lower border of the lesion and was facilitated by the endoscopic cap.

dyspepsia in two patients and dysphagia to solid food in the other two. On imaging, the tumor appeared as a C-shaped lesion originating from the posterior aspect of the gastroesophageal junction and involving between $50 \%$ and $75 \%$ of the circumference of the cardia. On endoscopic ultrasound examination, the tumor appeared

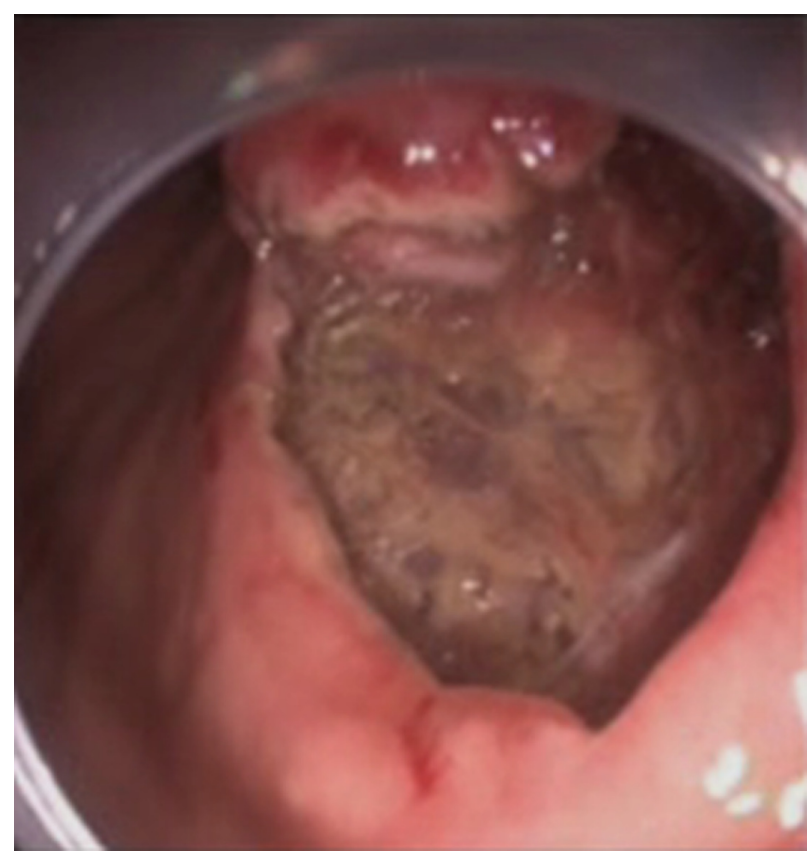

Figure 3 Aspect of the mucosal defect in the subcardial region before application of the endoclips. 


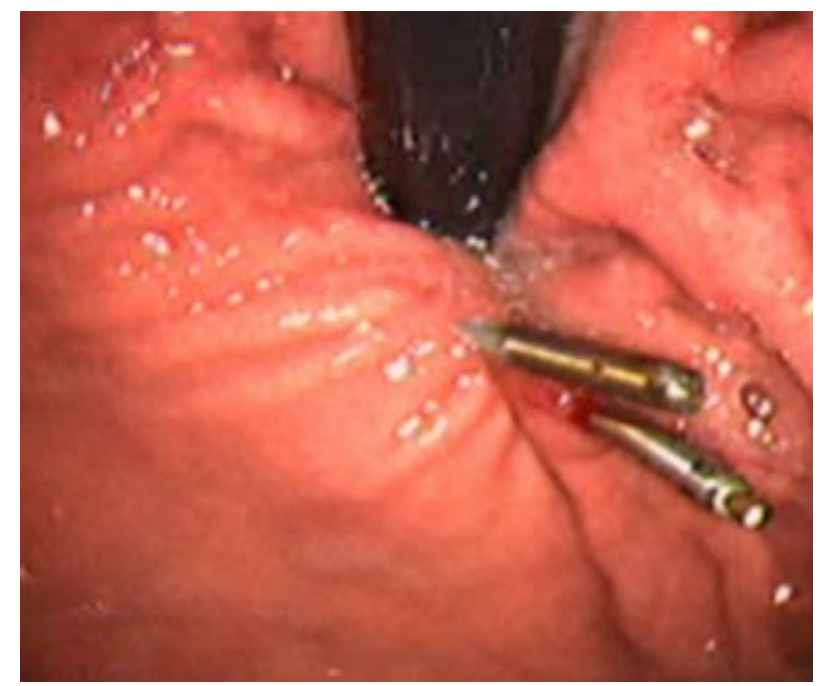

Figure 4 Aspect of the subcardial region after application of the endoclips.

to originate from the muscolaris propria in three patients and from the muscolaris mucosa in one.

The mean operative time was 180 minutes (range 150-220 minutes). No bleeding occurred during the procedure. Fragmentation of the tumor in the gastric cavity was necessary in the third patient in order to ease transoral retrieval. All patients returned to the surgical ward after the procedure.

The complete $(\mathrm{R}-0)$ resection rate was $100 \%$. The mean size of the removed lesions was $6.5 \mathrm{~cm}$ (range $4-12 \mathrm{~cm}$ ) and the mean thickness $2 \mathrm{~cm}(2-4 \mathrm{~cm})$ (Figure 5).

Pathological examination confirmed the diagnosis of leiomyoma in all cases. Immunohistochemical analysis showed negativity for desmin, sm-actin, and a CD117 (c-kit) (Table 1).

No complications were observed except for some degree of air filtration causing symptomatic pneumoperitoneum

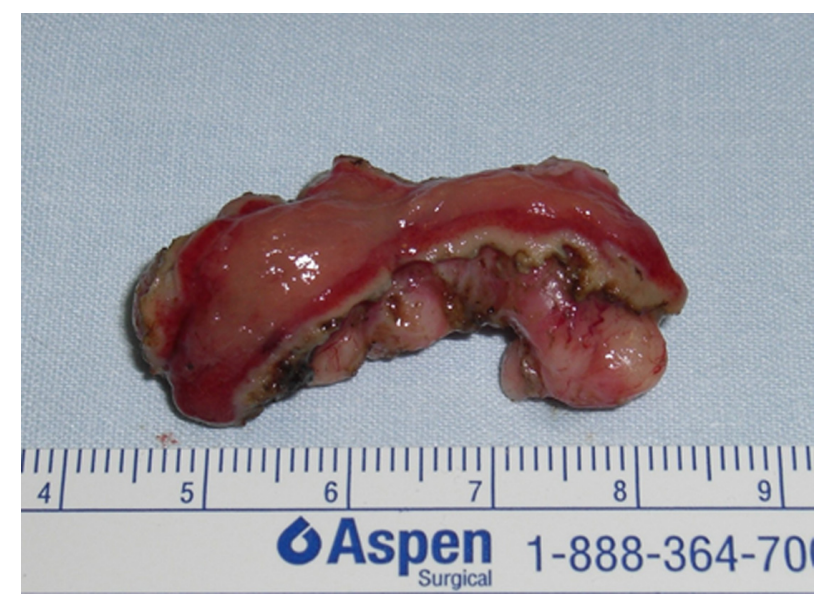

Figure 5 Operative specimen after endoscopic enucleation. The overlying mucosa was resected en bloc.

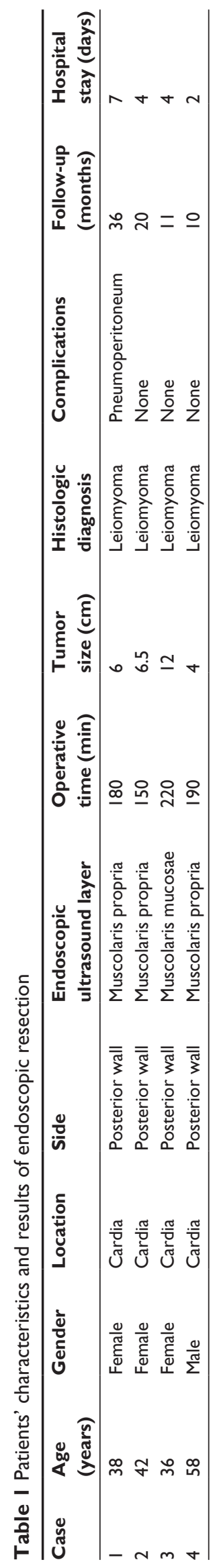


in the first patient of the series. This patient complained of a severe and persistent abdominal pain just at the end of the endoscopic procedure. Physical examination revealed generalized distension and tenderness of the abdomen, and a plain film revealed free air in the peritoneal cavity. A paracentesis using a 20-gauge needle catheter was performed, and about 3 liters of air was drained from the peritoneal cavity, with complete symptom resolution. A gastrographin swallow study was performed on the first postoperative day and did not show leakage at the gastroesophageal junction. A prophylactic paracentesis at the end of the endoscopic procedure was performed in the other three patients to prevent this complication. In all individuals, only a moderate subcutaneous emphysema developed and spontaneously resolved in about 3 days.

All patients were allowed to drink clear liquids 24 hours after the procedure and were then moved to a soft diet. Length of hospital stay ranged from 2 days to 7 days. All patients were discharged on proton pump inhibitor therapy. Follow-up endoscopy was performed 2-3 months after the operation in order to assess healing. No evidence of residual lesion was seen, and some of the endoclips were still attached to the mucosa. All patients were relieved of symptoms and satisfied of their postoperative status at a mean follow-up of 19.2 months (range 10-36 months).

\section{Discussion}

The technique of upper gastrointestinal submucosal tumor enucleation using an insulated-tip electrosurgical knife was first described by Park et $\mathrm{al}^{9}$ in 2004 . We applied the same basic endoscopic technique to patients with larger tumors originating from the posterior wall of the gastroesophageal junction. Due to the significant size of the tumor and a longer mucosal incision, we used clips to close the mucosal defect. Endoscopic removal of these giant leiomyomas of the gastroesophageal junction was feasible and safe in our experience. Pneumoperitoneum is a potential complication of the submucosal dissection at the posterior aspect of the gastric cardia where the serosal layer is lacking. Filtration of air through the thin posterior gastric layer was probably the cause of symptomatic pneumoperitoneum in one of our patients. ${ }^{10}$ Prophylactic paracentesis avoided postoperative abdominal distension in the other patients, but it did not prevent the appearance of mild subcutaneus emphysema.

Significant bleeding has also been reported after endoscopic enucleation and is related to the well-developed submucosal vascular network at the gastroesophageal junction. ${ }^{9}$ Closure of the mucosal defect with endoclips may have contributed to prevent postoperative bleeding in our patients.

The main limitation of this endoscopic technique is the long time required to perform the procedure with the currently available technology, even in the hands of expert operators. The procedure is best performed by two surgeons who are familiar with operative upper gastrointestinal endoscopy. The first operator is on the patient's left side and handles the scope; the second operator is at the patient's head and maintains the scope in a fixed position to prevent its malrotation and to disengage the right hand of the first operator. The monitor is placed at the upper left corner of the operating table. To optimize the retroflexed approach, it is convenient to start the incision at the lower border of the tumor. This provides a clear vision of the operative field without the obstructive effect of the mass, which, pushed by gravity, would make the dissection more difficult. In our opinion, the retroflexed approach is superior to the frontal one because it allows the operator to face the lesion from the gastric cavity and makes the dissection more comfortable by avoiding the flat working angle that forces the operator into a tangential use of the endoscopic instruments.

This small case series shows that the retroflexed endoscopic approach is feasible and safe for enucleation of even giant leiomyomas of the gastroesophageal junction. This avoids the need for open esophagogastrectomy or a combined endoscopic/laparoscopic transgastric approach. However, further clinical experience is necessary before the endoscopic technique can be established as the procedure of choice in these patients.

\section{Acknowledgments}

Conception and design: D Bona, L Bonavina; analysis and interpretation of the data: D Bona, A Aiolfi, D Bernardi; drafting of the article: D Bona, A Aiolfi, S Siboni, L Bonavina; critical revision: L Bonavina; final approval: all authors.

\section{Disclosure}

The investigation was conducted in conformity with clinical and ethical principles of research. There have been no commercial associations that might pose a conflict of interest in connection with the manuscript.

\section{References}

1. Appelman HD, Helwig EB. Gastric epithelioid leiomyoma and leiomyosarcoma (leiomyoblastoma). Cancer. 1976;38:708-728.

2. Lee Mg, Lim JS, Kwon JE. Gastric true leiomyoma: computed tomographic findings and pathological correlation. J Comp Assist Tomogr. 2007;31: 204-208. 
3. Jeong ID, Sung SW, Bang SJ. Endoscopic enucleation for gastric subepithelial tumors originating in the muscularis propria layer. Surg Endosc. 2011;25:468-474.

4. Shim CS, Jung IS. Endoscopic removal of submucosal tumors: procedure diagnosis, technical options, and results. Endoscopy. 2005;37: 646-654.

5. Bonavina L, Segalin A, Rosati R, et al. Surgical therapy of esophageal leiomyoma. J Am Coll Surg. 1995;181:257-262.

6. Shim JH, Lee HH, Yoo HM, Jeon HM. Intragastric approach for submucosal tumors located near the Z-line: a hybrid laparoscopic and endoscopic technique. J Surg Oncol. 2011;104:312-315. Epub April 4, 2011.
7. Tagaya N, Mikami H, Kogure H. Laparoscopic intragastric stapled resection of gastric submucosal tumors located near the esophagogastric junction. Surg Endosc. 2002;16:177-179.

8. Nguyen NT, Jim J, Nguyen A. Laparoscopic resection of gastric stromal tumor: a tailored approach. Am Surg. 2003;69:946-950.

9. Park YS, Park SW, Kim TI. Endoscopic enucleation of upper-GI submucosal tumors by using an insulated-tip electrosurgical knife. Gastrointest Endosc. 2004;59:409-415.

10. Siboni S, Bona D, Abate E, Bonavina L. Tension pneumoperitoneum following endoscopic submucosal dissection of leiomyoma of the cardia. Endoscopy. 2010;42 Suppl 2:E152. Epub June 16, 2010.

\section{Publish your work in this journal}

Clinical and Experimental Gastroenterology is an international, peerreviewed, open access journal, publishing all aspects of gastroenterology in the clinic and laboratory, including: Pathology, pathophysiology of gastrointestinal disease; Investigation and treatment of gastointestinal disease; Pharmacology of drugs used in the alimentary tract;
Immunology/genetics/genomics related to gastrointestinal disease. This journal is indexed on CAS. The manuscript management system is completely online and includes a very quick and fair peer-review system. Visit http://www.dovepress.com/testimonials.php to read real quotes from published authors.

Submit your manuscript here: http://www.dovepress.com/clinical-and-experimental-gastroenterology-journal 